\title{
Technologies to support the railway circulation in emergency conditions
}

\author{
G. Sciutto ${ }^{1}$, M. Lucchini ${ }^{2}$, D. Mazzini ${ }^{2} \&$ C. Veglia ${ }^{3}$ \\ ${ }^{1}$ SCIRO S.p.A., Via A. Gavoitt 5/6, Genova, Italy \\ ${ }^{2}$ CIELI - Centro Italiano di Eccellenza sulla Logistica Integrata, \\ Via Bensa 1, Genova, Italy \\ ${ }^{3}$ DIE - Dipartimento Ingegneria Elettrica, \\ Università degli Studi di Genova, Via Opera Pia 11a, Genova, Italy
}

\begin{abstract}
The issue of the modal rebalance has concentrated the attention of European countries on the standardization of the railway system in order to allow convoys to travel more simply around all of Europe. It has been reached as a result of the creation of international work groups, the realization of ERTMS/ETCS System (European Railway Traffic Management System/European Traffic control System) and of the relative technical specifications of interoperability.

The possibility of applying radar based technologies to ERTMS has been estimated for technologies able to improve the visibility of objects on the track and to bypass the geometric distortion problem in the most complete possible way.

Moreover, the radar based technology concurs, if compared to the optical based devices that use cameras, to exceed the problems concerned in the reduction of visibility for atmospheric causes.

The objective of the study program is to estimate the possibility to apply, in particular to the High Speed system characterized by a high speed rank and by a line architecture that is quite simple and linear, resorting to a simulation instrument which will concur, opportunely shaped with different scenes, to estimate if it could also be applied to different regimes of circulation.
\end{abstract}

Keywords: ERTMS, ETCS, radar, on sight, accident, railway network, safety, technology, trackside subsystem, trainborne subsystem. 


\section{Introduction}

The existing state-of-the-art does not include any on-the-move optical recognition and identification system for use on-board trains, nor any automated active intervention on the train movement to prevent collision with obstacles lying on the track.

Currently, this aspect of safety relies on human observation by the driver, or prior information. There is in no real time observation through optical or other means. Automated braking is triggered only when crossing the wrong signalling signs.

\section{Application technology to ERTMS/ETCS system}

The content of this document explains how technology can be utilized for support to railway safety. The application of new technologies is necessary for a greater exploitation of railway transport, as indicated from European Community politics [1]. The modal re-balance is the unique instrument for adaptation to increases in transport.

Actually, the application of new technology instruments to railway transport, permits the increase of potentiality as well as of safety for this transport. In particular, the concept of safety for railway transport, is defined from D.P.R. $753 / 1980$.

This legal provision describes the aim of the installation of new technologies, which is to avoid railway incidents and to eliminate fatality accidents. In particular this technology is utilized in the "on sight" movement.

The issue of the modal rebalance has concentrated the attention of European countries on the standardization of the railway system in order to allow the convoys to travel more simply around Europe. It has been reached as a result of the creation of international work groups, the realization of ERTMS/ETCS System (European Railway Traffic Management System/European Traffic control System) and of the relative technical specifications of interoperability.

A railway line can be qualified as interoperable when it's equipped (e.g. with the ERTMS) in such a way to allow the transit of convoys coming from either various producers or various countries, not requiring the substitution of the engine and of the driver.

ERTMS system is based on different operating modes corresponding to different allocations of responsibility between the trackside subsystem (SST) that sends all the information to the driver for the conduct of the train, and the trainborne subsystem (SSB), that elaborates the information automatically and regulates the speed of the convoy in a safe way.

The operating mode correspondent to the total allocation of the responsibility to the SST is called 'Full Supervision'; in such conditions the system gives the train all the necessary information for the control of the movement.

Opposite this is the operating modality defined On Sight that is a consequence of a degraded condition, characterized by a lack of the availability of the 
information concerning the state of the way, in which the train staff are responsible for ensuring that the way is free.

From the technological point of view, system ERTMS is able to manage the Full Supervision modality in emergency conditions, and support the driver, in the absence of technologies, for the On Sight movement phase.

Currently, technologies based on various kinds of sensors are being applied in the automotive field, characterized by usage conditions radically different from the railway field where there is a risk of a greater magnitude because of the reduced wheel/track friction and of the consequent greater space of arrest in case of emergency stop. The possibility to apply radar based technologies to ERTMS has been estimated, with technologies able to improve the visibility of objects on track and to bypass the geometric distortion problem in the most complete possible way.

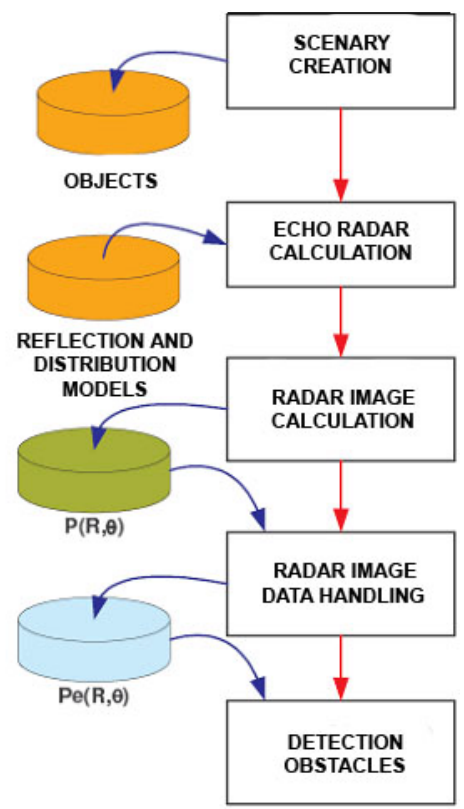

Figure 1: $\quad$ Simulation of the scenery.

\section{Radar system}

In compliance with obstacle detection functions, the simulation process utilized is compound at some essential passages [3]:

- Generation of the scenery;

- Calculation of the radar signal;

- Calculation of the radar image;

- Radar image data handling;

- Objects recognition. 


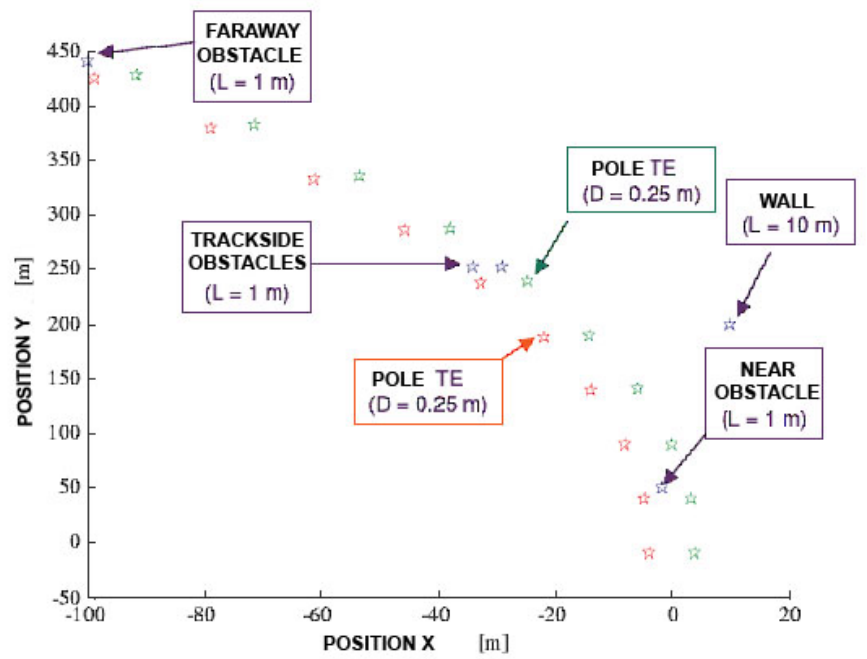

Figure 2: $\quad$ Generation of the scenery.
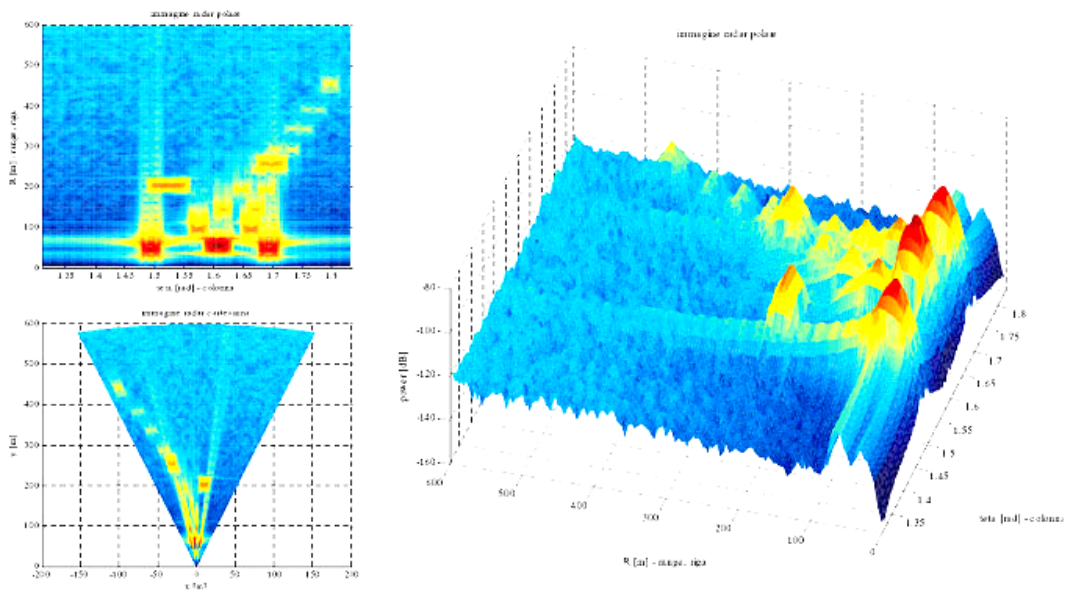

Figure 3: Decoded radar image.

For example, the scenery generation is created with TE poles, a wall and some obstacles on the trackside.

The decoded radar image in the simulation could be depicted using different kinds of reference systems, as polar, cartesian and two/three dimensional [2]. After the imaging process, the final result reached is the scenario graph that tracks down the present objects.

The geometric distortion is a phenomenon connected to the concept of perspective sight which exceeds a certain distance (typically of $200 \mathrm{~m}$ ), where 
objective difficulties in the appraisal of the effective distances are met, difficulties that become greater if the trackside poles, or other external elements, can't be used to advantage by the driver to understand the real distances.

Moreover, the radar based technology concurs, if compared to the optical based devices that use cameras, to exceed the problems concerned with the reduction of visibility for atmospheric causes.

The objective of the study program is to estimate the possibility to apply, in particular to the High Speed system, characterized by a high speed rank and by a line architecture that is quite simple and linear, resorting to a simulation instrument which will concur, opportunely shaped with different scenes, to estimate if it could also be applied to different regimes of circulation.
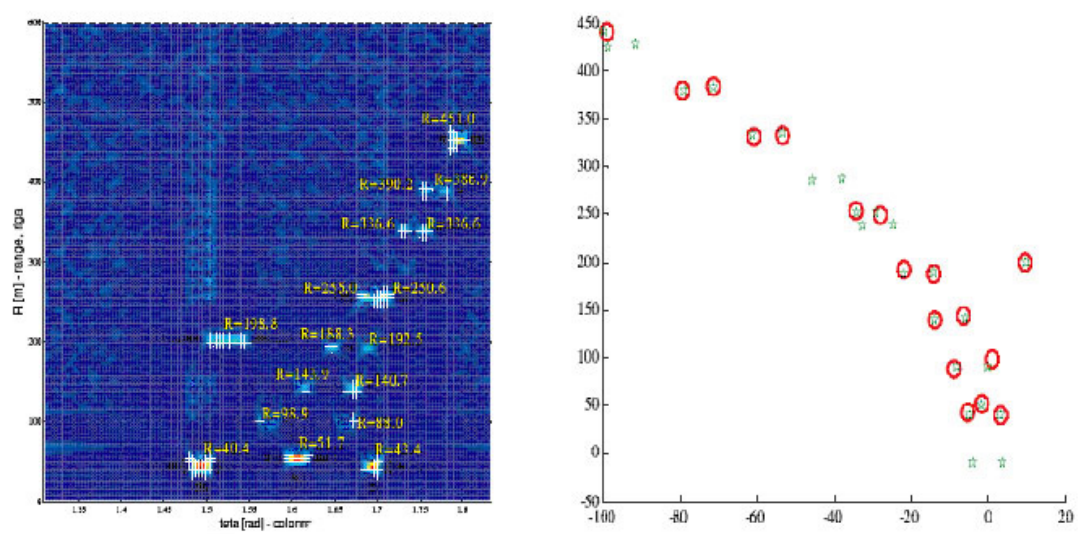

Figure 4: Objects recognition (cluster).

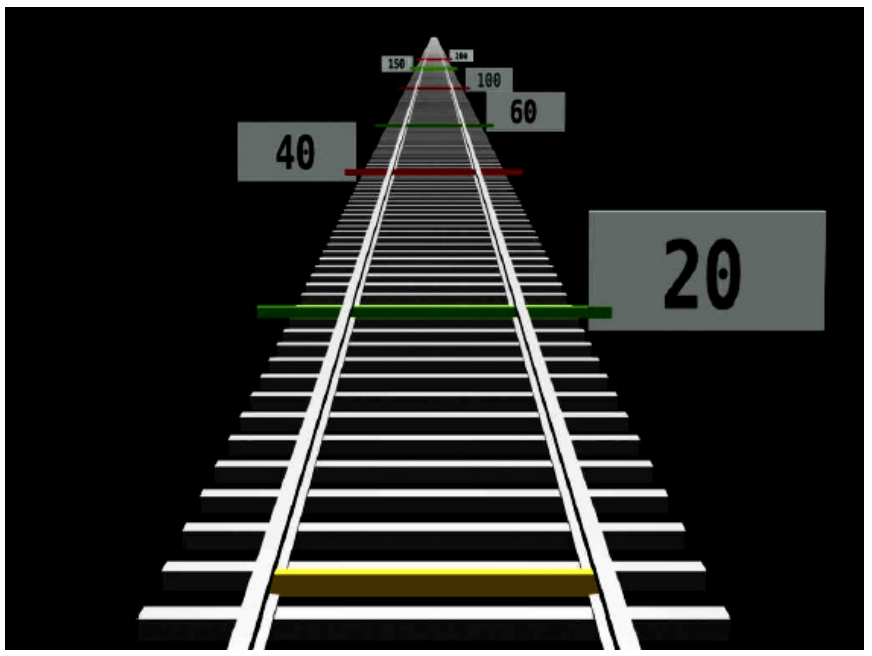

Figure 5: The geometric distortion. 


\section{Conclusion}

A further research development will be based on further simulations and real applications taking into account various scenarios.

Application limits - especially in the various circulation conditions of ERTMS system - will be evaluated after the last phase of in field tests starts.

Development of the research activity:

- Simulations Implementation;Different Scenario Application;

- Evaluation of the System Applicability;

- Indications on the possible application under different circulation conditions;

- $\quad$ Testing on Real Field.

\section{References}

[1] G. Sciutto, M. Galaverna - Tecnologie dei Trasporti e Territorio, 2000.

[2] Bassem R. Mahafza - Radar Systems Analysis and design using Matlab, 2000.

[3] Merrill I. Skolnik - Introduction to Radar Systems, 2001. 